\title{
Perspective
}

PERSPECTIVE Actualité en histoire de l'art

1 | 2016

Textiles

\section{Les textiles à la période moderne : circulation, échanges et mondialisation}

Une discussion entre Maria João Ferreira, Liza Oliver et Corinne

Thépaut-Cabasset, menée par Maria Ludovica Rosati

Textiles in Early Modern Period: Circulation, Trade and Globalization

Maria João Ferreira, Liza Oliver, Maria Ludovica Rosati et Corinne ThépautCabasset

Traducteur : Françoise Jaouën et Monique Le Moing

\section{(2) OpenEdition}

Journals

Édition électronique

URL : http://journals.openedition.org/perspective/6287

DOI : 10.4000/perspective.6287

ISSN : 2269-7721

Éditeur

Institut national d'histoire de l'art

Édition imprimée

Date de publication : 30 juin 2016

Pagination : 21-32

ISBN : 978-2-917902-31-8

ISSN : $1777-7852$

Référence électronique

Maria João Ferreira, Liza Oliver, Maria Ludovica Rosati et Corinne Thépaut-Cabasset, « Les textiles à la période moderne : circulation, échanges et mondialisation », Perspective [En ligne], 1 | 2016, mis en ligne le 31 décembre 2016, consulté le 01 octobre 2020. URL : http://journals.openedition.org/ perspective/6287 ; DOI : https://doi.org/10.4000/perspective.6287 


\title{
Les textiles à la période moderne : circulation, échanges et mondialisation
}

\author{
Une discussion entre Maria João Ferreira, \\ Liza Oliver et Corinne Thépaut-Cabasset, \\ menée par Maria Ludovica Rosati
}

Dans les textes publiés récemment dans le domaine des textiles, on rencontre fréquemment le terme global associé à divers titres à ce champ de recherches ${ }^{l}$. Le besoin d'inscrire les études sur le textile dans une globalité au sein de systèmes mondiaux ne répond pas seulement à la tendance qui se généralise aujourd'hui dans la réflexion critique et cherche à adopter une perspective transculturelle et mondiale; il semble également dicté par la nature particulière du textile en tant que medium. À travers l'histoire, et plus particulièrement depuis le début de la période moderne, le textile peut être considéré comme le medium mondial par excellence.

$A u$ début de la période moderne, c'est tout le système de production qui prend une ampleur planétaire: depuis l'approvisionnement en matières premières jusqu'au développement des techniques, en passant par la genèse des motifs, la quasi-totalité des manufactures locales s'inscrivent dans un scénario élargi reposant sur les contacts, les migrations et les échanges avec d'autres réalités, et caractérisé par de multiples interdépendances. À cette époque, le commerce des textiles est pareillement d'ordre planétaire. On connaissait déjà, à la période pré moderne, des exemples de liens supranationaux et interculturels, et on sait que certaines productions textiles étaient destinées à l'exportation, mais c'est au début de l'ère moderne que le réseau des échanges acquiert progressivement une dimension véritablement mondiale, ce qui influe sur les manufactures et la consommation. On peut également considérer comme mondiale la propagation de certains phénomènes culturels, depuis la large circulation des textiles jusqu'à l'usage qui en est fait, notamment une tendance à leur octroyer un contenu sémantique particulier ou à les adopter dans le cadre de pratiques sociales particulières, ou encore l'essor de la mode et le goût pour l'exotisme et l'altérité.

On peut même considérer que le textile, en tant que champ d'investigation, possède une dimension globale, car il offre de multiples perspectives de recherches qui s'entrecroisent; il fait intervenir des questions économiques, artistiques, technologiques, politiques, sociales et culturelles, au point que l'approche interdisciplinaire est sans doute souvent la meilleure clef méthodologique permettant de les appréhender. Par le biais du textile, il devient possible d'explorer des phénomènes humains de dimension complexe. [Maria Ludovica Rosati]

Maria Ludovica Rosati. Au début de la période moderne, les textiles circulent non seulement au sein d'un système planétaire, mais certaines formes d'échange, telles que la pratique diplomatique consistant à offrir des étoffes précieuses, contribuent

\footnotetext{
Maria João Ferreira est chercheur intégré au CHAM Centre portugais pour I'histoire globale - FCSH/ NOVA-UAC; elle étudie la consommation textile au Portugal au cours de l'époque moderne, en particulier la production textile chinoise pour le marché portugais.
}

Liza Oliver est Assistant Professor of Art History and South Asia Studies au Wellesley College. Ses recherches portent sur les échanges matériels et intellectuels entre

I'Asie du Sud et l'Europe du XVII ${ }^{e}$ au XIXe siècle.

Après avoir obtenu son doctorat en histoire de l'art (Scuola Normale Superiore,

Pise), Maria Ludovica Rosati a travaillé à la Scuola Normale, à l'université de Trieste, au Museo del Bargello

(Florence), au Museo del Tessuto (Prato), et participé à diverses expositions sur les textiles anciens. Elle coordonne les recherches sur les vêtements sacerdotaux de Benoît XI à Pérouse, un projet financé par la Bruschettini Foundation for Islamic and Asian Art de Gênes.

Corinne Thépaut-Cabasset, historienne, est spécialiste des arts décoratifs des débuts de I'ère moderne. Chercheur 
associé au Victoria and Albert Museum de Londres, elle a participé au projet européen HERA baptisé «Fashioning the Early Modern, Creativity and Innovation in Europe: 1500-1800» (2010-2013). Elle est chargée de recherche au château de Versailles, et titulaire d'une bourse Marie Skłodowska-

Curie à I'université de Copenhague.

1. Artiste indien anonyme, Noble Portugaise en Inde, extrait du Codex Casanatense, vers 1540-1550, Rome, Biblioteca Casanatense, ms 1889, fol. 97.

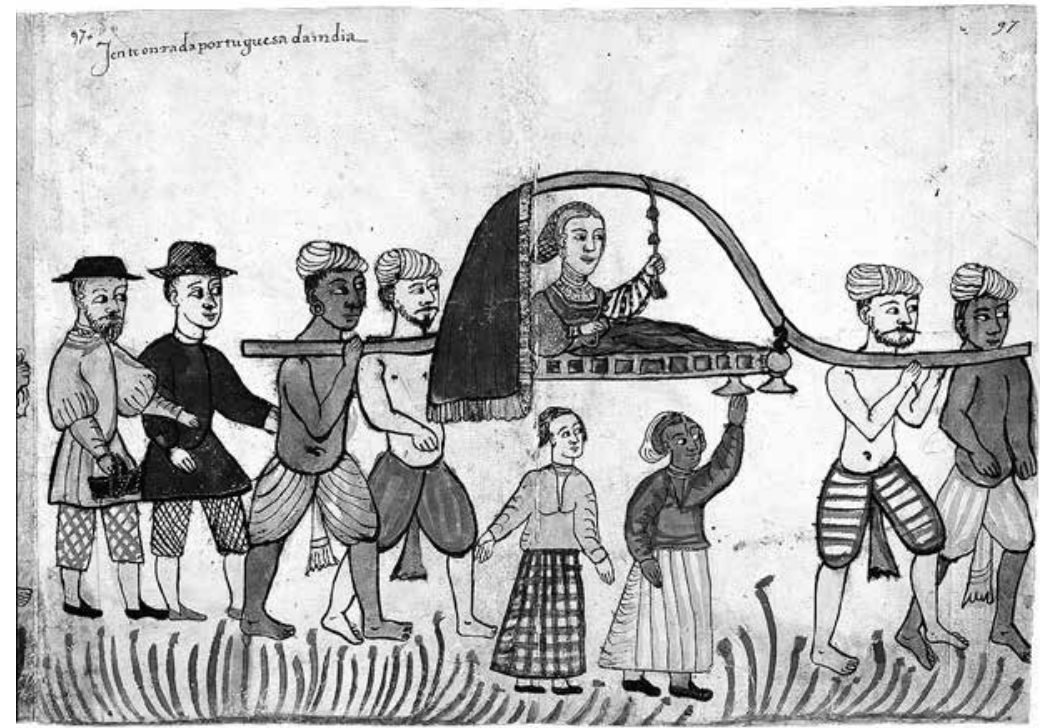
des relations entre individus? attiraient l'attention par leur magnificence. à créer un terrain commun entre différentes cultures, terrain où se partagent aussi les significations de ces artefacts. Peut-on alors considérer le medium textile comme un outil permettant de bâtir une identité, une image de soi et des autres, ainsi que

Maria João Ferreira. Le rôle joué par les textiles, à la fois comme marchandises et agents indispensables à la mise en scène et à la promotion du pouvoir, dans la stratégie économique et géopolitique des Portugais en Asie, et après l'arrivée de Vasco de Gama en Inde en 1498, est particulièrement intéressant. Confrontés au degré élevé de sophistication culturelle et commerciale des sociétés locales, et déterminés à construire une Asie portugaise, les Portugais durent apprendre à s'adapter à cette réalité et à la connaître profondément s'ils voulaient triompher. Cela impliquait une " asiatisation » des pratiques diplomatiques et des rapports humains. Ils se laissèrent influencer par les traditions autochtones qu'ils adoptèrent et intégrèrent en grande partie, parce qu'ils y trouvaient un écho ou une raison d'être qui servait leurs propres intérêts, tant dans l'espace ultramarin que dans le Royaume (fig. 1). Ce n'est pas un hasard si les chroniqueurs de l'époque accordent une attention particulière au protocole complexe et au faste des souverains locaux auxquels concourent les ornements textiles et leur richesse matérielle, chromatique et décorative. Le roi dom Manuel ler s'appropria le modèle à des fins personnelles en copiant la splendeur asiatique lors de ses grandes démonstrations publiques et celles de ses représentants, comme en témoigne l'impressionnante délégation qu'il envoya au pape Léon $X$ en $1514^{2}$. Une ambassade en tous points orientale, tant dans l'ostentation des richesses que dans l'exhibition des hommes et des animaux de ces régions. Les textiles asiatiques utilisés pour les habits sophistiqués, les accessoires qui agrémentaient le défilé et l'ornement liturgique offert au pontife

L'acquisition et l'exhibition des textiles extra-européens donnent corps et portée, en plusieurs sens, au projet ultramarin portugais. Assimilés par le quotidien portugais, ces objets présentent un intérêt et matérialisent une accumulation de connaissance acquise au contact de l'autre ${ }^{3}$, de la même façon qu'ils deviennent des symboles du pouvoir impérial portugais dans le monde. Ils aident à fixer la mémoire d'un Orient portugais qui est à la fois une réalité et une représentation, et surtout un puissant agent de propagande extérieure, si nécessaire à la réhabilitation de l'image du Portugal en Europe pendant le XVII siècle $^{4}$. En 1669, décors chinois et tapis persans ornaient le Palais royal lors du baptême de la princesse Isabelle de Bragance. La même année, dom Pedro, le prince régent, offrait des pièces de soie de Chine à Cosme III de Médicis lors du passage de celui-ci à Lisbonne. Pour des raisons de goût, mais surtout de prestige et d'ostentation, les textiles asiatiques participent désormais des mises en scène montées à l'occasion d'événements solennels et deviennent partie intégrante des présents diplomatiques que la Couronne portugaise offre à ses homologues, même quand l'empire se trouve en phase de déclin. 
Liza Oliver. Le contexte sud-asiatique fournit un éclairage intéressant sur le rôle des étoffes dans le cadre diplomatique et dans l'établissement de relations. Comme l'ont montré Philip Wagoner et Finbarr Barry Flood, les étoffes et le costume constituent un élément crucial des échanges matériels qui alimentent les relations entre hindous et musulmans à l'époque médiévale ${ }^{5}$. Ils révèlent les négociations et les mélanges continus d'identités qui s'opèrent entre les deux religions et les diverses cultures qui les composent. Si le tissu est au cœur de ces négociations, c'est parce qu'il occupe une place singulière
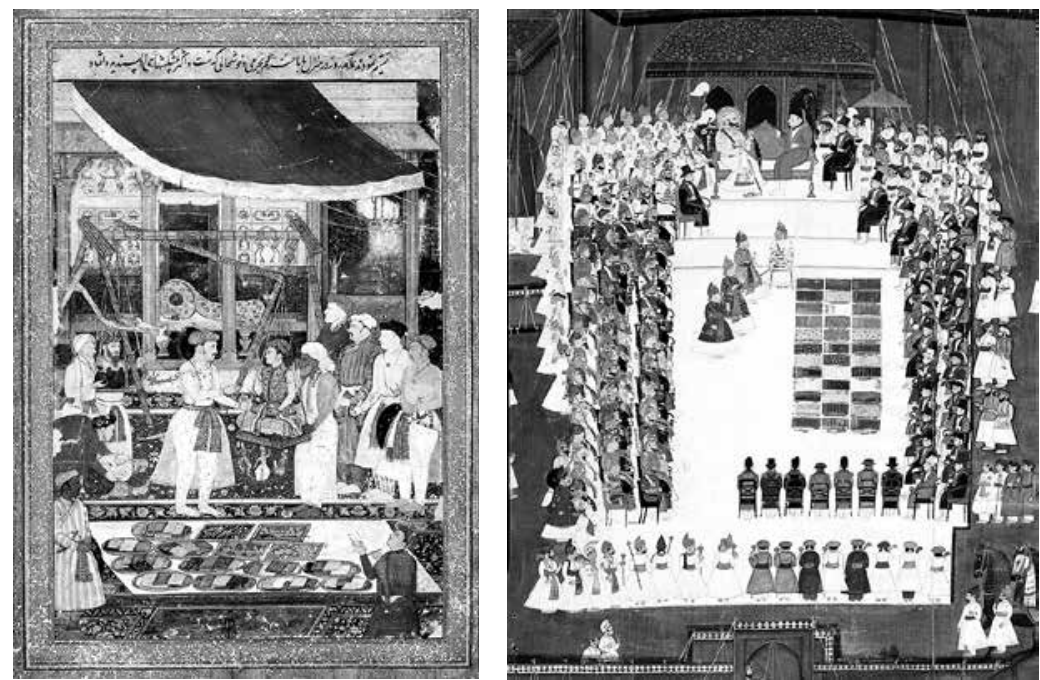

dans toutes les religions et cultures

sud-asiatiques; on lui attribue en effet la capacité de transmettre une valeur à la fois matérielle et symbolique et d'absorber les qualités de celui qui l'a fabriqué, qui le porte ou le possède ${ }^{6}$. L'étoffe joue ainsi depuis longtemps un rôle central dans certaines pratiques, comme la puja (rituel) des hindous, ou encore la cérémonie de la khil'a (robe d'honneur) des Moghols. Dans la miniature de Jahángir (fig. 2), les robes sont mises en valeur au premier plan car elles sont le signe de la richesse matérielle de l'empereur et, offertes en cadeau, elles incorporeront l'aura et l'esprit du souverain dans l'existence de celui qui les portera.

Les textiles contribuent largement à créer un espace commun entre peuples et cultures, mais ils peuvent aussi dévoiler de profonds malentendus. Si les Européens en Inde se joignent aux pratiques du don des cours royales sud-asiatiques afin de nouer des alliances et d'obtenir des droits commerciaux, ils se méprennent souvent en y voyant non pas un honneur ou un hommage mais une forme de corruption. Le tableau de Ghasi représentant le darbar (audience officielle) de 1832 entre Lord Cavendish Bentinck et Jawan Singh, maharana du Mewar (fig. 3) montre comment l'évolution des structures politiques et de la dynamique de pouvoir peut entraîner des incongruités sur la signification des artefacts. L'image illustre le moment où les présents du souverain (des rouleaux d'étoffes multicolores) sont remis au Britannique. Jawan Singh adapte ici une longue tradition de cadeaux princiers aux nouvelles exigences d'une emprise coloniale croissante. Mais ces étoffes, offertes en signe d'alliance symbolique avec les Britanniques, prennent souvent une toute autre signification à leur arrivée en Angleterre. Au milieu du XIX siècle, la reine Victoria considère que le port de ces étoffes fait injure aux fabricants britanniques. Elle met en avant la production locale en commandant à une manufacture londonienne un rouet avec lequel elle se fait photographier à plusieurs reprises. On voit ainsi comment, à l'époque, les Anglais tendent à envisager l'étoffe exclusivement en termes de plus-value économique et matérielle apportée à la nation, et non sous la dimension symbolique que lui accordent les cours princières sud-asiatiques qui ont coutume d'en faire présent.

Corinne Thépaut-Cabasset. Un document exceptionnel conservé au ministère des Affaires étrangères à Paris nous informe sur les quantités phénoménales d'étoffes (de soie et de laine) sorties des manufactures royales et offertes par Louis XIV aux princes, souveraines et ambassadeurs au cours de son règne ${ }^{7}$. II montre que la pratique des cadeaux sert essentiellement à faire circuler les produits français (dont les textiles) et à promouvoir
2. Jahángír Weighs Prince Khurram against Gold and Silver Uahángír pèse le prince Khurram avec de l'or et de l'argent], extrait de Túzuki-i Jahángírí [Mémoires de l'empereur Jahángír], vers 1615, Londres, The British Museum.

3. Ghasi, Maharana Jawan Singh of Mewar Receiving the Governor General of India, Lord William Cavendish Bentinck, February 8th, 1832 [Le maharana Jawan Singh du Mewar reçoit le Gouverneur général de l'Inde, Lord William Cavendish Bentick, le 8 février 1832], détail des textiles colorés offerts en présents diplomatiques, vers 1832, New York, Brooklyn Museum, inv. 2002.34. 


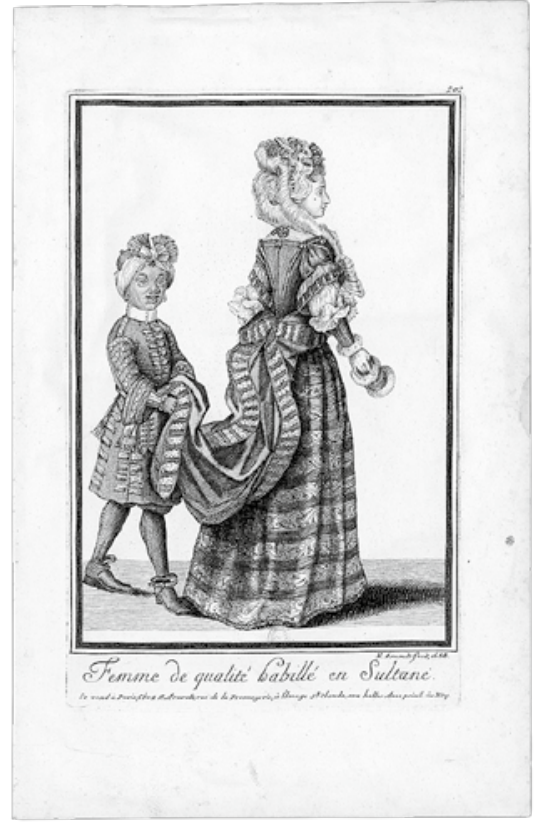

I'innovation française à travers le monde. Il souligne également l'attrait de ce medium et l'usage qui en est fait pour construire et diffuser à la fois une image de soi et une image des autres.

Un autre aspect est révélé par les correspondances et les rapports diplomatiques, qui offrent des exemples frappants de circulation des textiles et des modes à la fois en Europe et au-delà.

Dans une étude portant sur un agent bavarois basé à Paris à la fin du XVII siècle, on peut examiner les commandes adressées par le Prince électeur Maximilien Emmanuel et les documents issus des douanes françaises autorisant les " ballots » de textile à sortir du pays ${ }^{8}$. Les archives nationales bavaroises ont en effet conservé un ensemble unique de plusieurs centaines de missives d'un Bavarois résidant à Paris et adressées au Prince électeur de Bavière, qui concerne pour une large part les acquisitions faites à Paris pour le prince et sa cour. La principale fonction de leur auteur était d'acheter des pièces d'habillement françaises pour la garde-robe princière. Cette correspondance montre combien de pièces ont été envisagées, choisies, achetées et enfin expédiées à Munich. Rédigées entre 1674 et 1689, elles fournissent de précieux renseignements sur les marchands et les produits disponibles à Paris, considéré à l'époque comme la capitale incontestée de la mode européenne' 9

Certains documents peu étudiés tels que les systèmes modernes

4. Nicolas Arnoult, Femme de qualité habillé [sic] en sultane, 1688, Paris, BnF, département des Estampes et de la Photographie. de taxation présentent également un grand intérêt. C'est notamment le cas de ceux ayant trait aux droits de douane appliqués à Madrid au XVIII siècle, des documents qui montrent le schéma de circulation et de consommation des textiles (entre autres produits) en provenance de pays étrangers ${ }^{10}$.

\begin{abstract}
Maria Ludovica Rosati. Dans des situations historiques et géographiques différentes, on observe souvent un processus d'adaptation des textiles étrangers à de nouvelles formes d'usage spécifiques au contexte d'adoption. Les étoffes asiatiques sont par exemples utilisées en Europe pour les vêtements sacerdotaux; depuis l'époque d'Edo, les tissus persans et indiens sont réservés à la cérémonie du thé. Peut-on alors considérer que cette forte adaptabilité du medium textile est à la fois une conséquence de son caractère planétaire et une précondition de sa mondialisation? Comment expliquer ce processus d'adaptation du point de vue des acteurs impliqués?
\end{abstract}

Corinne Thépaut-Cabasset. La mode semble avoir fluctué très rapidement, suivant le cours des événements de la cour et de la ville. Le Mercure galant, périodique consacré aux nouvelles de Paris et de la cour (1672-1711), comportait une rubrique portant sur les nouvelles modes et tendances ${ }^{11}$, dans laquelle on découvre la grande diversité des termes employés pour décrire les textiles et le style vestimentaire de la fin du XVII siècle. La Chine, I'Inde et le Siam (actuelle Thaïlande) sont les nouvelles sources d'inspiration pour le commerce de la mode. Les manches "à la pagode", le taffetas " de la Chine », les robes "à la sultane » (fig. 4) ou "à la persane ", les éventails "à la siamoise » sont autant d'expressions qui entrent alors dans le vocabulaire français de la mode ${ }^{12}$.

Maria João Ferreira. Quand on analyse la contribution des textiles à la médiation et à la création de circuits globaux - autrement dit ce qui motive leur diffusion dans un cadre extraterritorial et la façon dont ils sont reçus dans d'autres contextes - de nombreux aspects doivent être pris en considération. Le cas que nous connaissons le mieux, celui 
des textiles chinois exportés vers le Portugal entre le XVle et le XVIII ${ }^{\mathrm{e}}$ siècle, en particulier ceux destinés à des fonctions liturgiques du culte catholique, illustre bien cette question $^{13}$. Nous nous référons à une manufacture originale dont la mise en pratique reflète bien les motivations et les référents artistico-culturels des deux royaumes impliqués et par conséquent la relation dialectique à la base de cette rencontre de civilisations.

Il en est de même d'une plateforme hybride de compréhension, révélatrice d'un sens aigu de l'opportunité et de l'initiative de la part de celui qui produit et de celui qui acquiert. Stimulés par des intérêts économiques et commerciaux et donc poussés à conquérir de nouveaux marchés, les Chinois se révèlent suffisamment ingénieux pour adapter leur traditionnel programme technique, iconographique et plastique tout en s'adaptant eux-mêmes à de nouveaux procédés et à de nouvelles références en syntonie avec le goût et le désir des destinataires.

Cette posture et cette capacité d'adaptation, en grande partie à l'origine de la vitalité du secteur, se propagèrent vers d'autres centres manufacturiers asiatiques et se distinguèrent même comme des facteurs-clefs de la domination indienne en ce qui concernait la fourniture de textiles dans le monde entier jusqu'à la révolution industrielle occidentale ${ }^{14}$.

Si dans la perspective chinoise ces objets représentent surtout une opportunité commerciale, pour les Portugais leur fabrication fait aussi référence à des motivations d'un autre ordre. Dans ce processus de transferts successifs et de contextes renouvelés au fil du temps, les protagonistes et les champs d'action s'altèrent et les objets peuvent revêtir de nouvelles valeurs et de nouvelles significations. Différents et attractifs, véritables témoins matériels d'une Église qui se prétend universelle, ces objets rappellent le pouvoir politique de l'Église catholique dans le monde. Particulièrement en Asie, sous couvert du projet missionnaire sur lequel se fondait l'État de l'Inde lui-même - un État qui s'étendait d'Ormuz à Goa, Malaga, Macao et Nagasaki - par le biais du patronage portugais. Implicitement, utiliser ou exhiber des textiles chinois représente une forme d'émulation du savoir et du pouvoir impérial portugais dans ses multiples dimensions (économique, religieuse, politique, militaire, culturelle et artistique) ${ }^{15}$.

Liza Oliver. Je crois en effet que l'adaptabilité d'un textile - essentiellement due à sa portabilité, à sa valeur utilitaire et la tendance qui en découle à le commercialiser en gros - est une pré-condition de sa nature planétaire et un catalyseur majeur de la mondialisation en général. En ce qui concerne plus particulièrement l'Europe, l'étroite correspondance entre textiles et mondialisation ne peut être dissociée du commerce Atlantique des esclaves. Si l'on examine l'ensemble formé par l'adaptabilité, l'esclavage et la mondialisation, on peut affirmer en outre que les textiles sont également une précondition de la modernité. Selon la célèbre thèse de Paul Gilroy, l'esclave est la première figure véritablement moderne en Europe ; transnational en vertu de la définition même du "passage du milieu », le travail de l'esclave soutient les économies qui ont permis la dissémination à travers le monde des matériaux bruts et l'essor des marchés capitalistes ${ }^{16}$.

L'extraordinaire adaptabilité des textiles ressort nettement lorsque l'on considère que leur valeur fluctue d'un point de vue matériel, mais aussi en tant que symbole d'asservissement et en tant qu'objet d'agentivité, à $d^{\prime}$ innombrables points du spectre de l'existence de l'esclave. Les esclaves du « passage du milieu » étaient ainsi appelés parce qu'ils étaient achetés avec des textiles indiens et des imitations européennes. Mais comme le montre une miniature peinte sur un bouton, les sociétés antillaises font un usage complexe de ces mêmes textiles (fig. 5). L'adaptabilité se dévoile sur plusieurs registres dans cette miniature attribuée à l'artiste Agostino Brunias, actif au
5. Agostino Brunias (attr.), bouton peint provenant de Saint-Domingue (aujourd'hui Haïti), fin du XVIII ${ }^{e}$ siècle, New York, Cooper Hewitt, Smithsonian Design Museum.

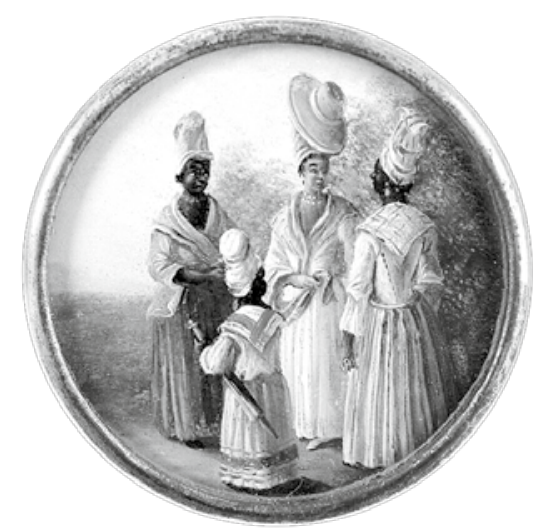




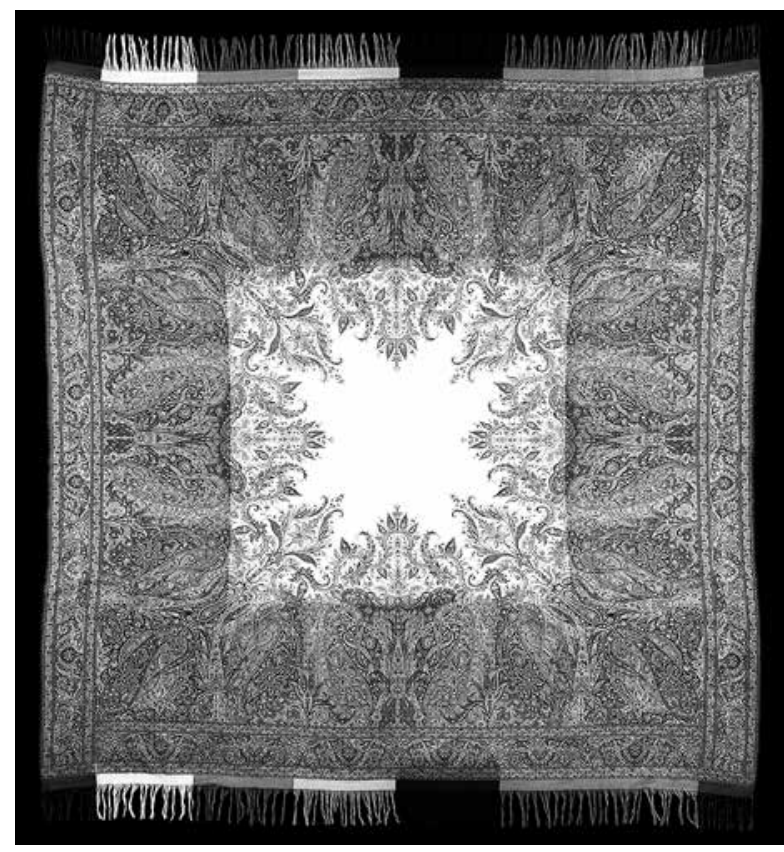

XVIII ${ }^{e}$ siècle. Elle montre des esclaves vêtus des textiles mêmes qui ont servi à les acheter, déstabilisant ainsi les hiérarchies sociales et remettant en question les oppositions possédant/possédé et sujet/objet sur lesquelles repose l'esclavage ${ }^{17}$. De surcroît, selon certaines sources sujettes à caution, ce bouton, avec environ dix-sept autres similaires attribués à Brunias, ornaient l'uniforme militaire de Toussaint Louverture, ancien esclave devenu chef de l'indépendance haïtienne. Cette histoire, même si elle est apocryphe, reste fascinante car elle semble indiquer que Louverture aurait ainsi transféré un agent d'asservissement dans une iconographie d'indépendance et d'autodétermination. De surcroît, ces boutons et l'uniforme auquel ils étaient, dit-on, attachés nous placent dans une proximité presque palpable avec un corps légendaire. Ornements de ce corps, ils sont des objets tactiles, intimes, et leur adaptabilité est de nature plus viscérale. J'y vois donc une réciprocité entre esclave et textile qui témoigne du rôle qu'ils jouent tous deux en tant qu'acteurs et agents de la mondialisation et de la modernité à laquelle elle a donné naissance.
6. Châle de laine à motifs paisley, probablement fabriqué en Écosse, 1840-1860, New York, Brooklyn Museum Costume Collection at The Metropolitan Museum of Art.

\begin{abstract}
Maria Ludovica Rosati. L'impact des textiles étrangers a joué un rôle, non seulement en incitant les manufactures locales à imiter et remanier les solutions exotiques à la production des étoffes, mais les motifs ont également migré d'une matière à l'autre, influant sur les goûts. Peut-on dire que le medium textile constitue un art majeur dans la création de systèmes ornementaux, dont certains sont peut-être communs à différentes civilisations?
\end{abstract}

Liza Oliver. Le boteh ou paisley (motif cachemire) représente le plein potentiel du rôle joué par les textiles dans la dissémination de systèmes ornementaux, avec ce que cela implique au niveau social. Le boteh figurant sur les châles en cachemire tissés au début du règne moghol au $\mathrm{XVI}^{\mathrm{e}}$ siècle montre de fortes similitudes avec des motifs floraux que l'on trouve sur d'autres media, comme par exemple les reliefs décoratifs de I'architecture moghole. Il serait intéressant de savoir quel medium a influencé l'autre, ou si, comme c'est probable, l'influence a été dialogique. Mais ce qu'il faut avant tout s'efforcer de découvrir, c'est pourquoi le boteh a connu sa plus grande évolution par le biais du textile, et comment ce medium a permis de diffuser le motif à travers le monde, pour en faire ce que l'on appelle aujourd'hui paisley.

Un châle en pashmînâ fabriqué en Europe au XIXe siècle et orné d'un motif cachemire reflète, de manière abstraite, de vastes structures et interactions sociales (fig. 6). Il témoigne tout d'abord de l'exploitation de la main-d'œuvre et des taxes écrasantes imposées aux tisseurs de pashmînâ auX XVIII e et XIX siècles dans le Cachemire sous domination afghane et sikh. C'est ce qui a permis au boteh, par l'intermédiaire du pashmînâ, de pénétrer massivement le marché européen et d'alimenter la demande d'imitations locales. Cette exploitation a également poussé les artisans miséreux du Cachemire à se soulever au XIXe siècle, un soulèvement qui fit de nombreuses victimes. Du côté européen, ce châle est à la fois le signe et le produit de nouvelles technologies - tel le métier jacquard - qui entraînent la dissémination rapide et à grande échelle des motifs, le développement et l'expansion de la législation sur la propriété dans 
le domaine visuel, et la stratification sociale, phénomènes accélérés par I'industrialisation. Ce contexte dévoile que le paisley n'est pas seulement un motif partagé entre l'Asie du Sud et l'Europe, mais un ensemble instable $d^{\prime}$ interactions et de conflits humains.

On peut ainsi dire, en effet, que les textiles sont sans aucun doute le fer de lance de la création et du partage de l'ornementation entre civilisations. Il faut cependant se garder de dissocier celle-ci de l'ensemble des transactions humaines sur lesquelles reposent sa dissémination, ce qu'il est aisé de faire lorsque l'on attribue trop d'agentivité au textile ou au motif lui-même. Les relations sociales sont toujours implicites dans les systèmes ornementaux. Lorsque l'on dit que des civilisations différentes « partagent » le même motif, qu'est-ce que cela signifie ? Le terme pourrait occulter des pratiques dynamiques, précisément situées dans le temps, et qui vont du vol au plagiat, de l'exploitation à l'adaptation, de l'altération au développement. Afin de ne pas diluer ces processus dans des débats génériques sur les questions d' « influence», il faut prendre en compte l'agentivité (ou l'absence d'agentivité) des acteurs humains impliqués dans ces processus de partage. En examinant les relations sociales inscrites dans ces symboles partagés, on se rendra sans doute compte qu'il n'existe aucune approche standard de la théorisation des processus de transmission. La révélation de ces spécificités devrait, selon moi, être l'objectif des études sur les partages de motifs ornementaux entre différentes cultures.

Corinne Thépaut-Cabasset. L'effet produit par la visite de la délégation siamoise à la cour de Louis XIV en septembre 1686 fournit l'exemple clef d'un tissu qui devint rapidement populaire, tout en évoluant au cours des cinquante années suivantes ${ }^{18}$. L'événement sensationnel que constituait cette visite diplomatique, couvert par les gazettes, suscita une grande curiosité pour les coutumes et les mœurs siamoises ${ }^{19}$.

Peu après la visite des ambassadeurs à Versailles et à Paris, les marchands de la capitale lancèrent un nouveau tissu baptisé "Siamoise ». Il s'agissait apparemment d'une étoffe légère à rayures faisant écho à celle portée par la délégation siamoise. On ne sait avec certitude si les fabricants français cherchaient à imiter un tissu originaire du Siam, ou s'ils s'en sont simplement inspirés pour leur création. En revanche, on sait de source sûre que, parmi les cadeaux envoyés par le roi de Siam à Louis XIV, se trouvaient différentes pièces d'étoffe (Mercure galant, 1686). C'est peut-être ce qui a incité d'astucieux marchands à créer un nouveau tissu français. Le terme "Siamoise », qu'il se réfère à la création française ou à un tissu siamois, est celui utilisé par les marchands dans la presse et sur les gravures de mode, et la nouvelle tendance est aussitôt lancée ${ }^{20}$.

En juin 1687, dans la rubrique de la nouvelle mode d'été, le Mercure galant annonce qu'un tissu à rayures multicolores baptisé "Siamoise ", connaît un grand succès chez les marchands. L'étoffe de laine, aussi douce et légère que la mousseline, est classée par les marchands parmi les "satins façonnés ». Autrement dit, elle rejoint les fibres communément utilisées dans la confection de mode : la laine, la soie et le coton $^{21}$.

En 1723, dans son Dictionnaire universel de commerce..., Jacques Savary des Bruslons mentionne cette étoffe, faite de soie et de coton, et note qu'elle est apparue en France pour la première fois à l'occasion de la visite des ambassadeurs du Siam. En 1736, les manufactures royales continuent à produire en Normandie une cotonnade à rayures ou à carreaux appelé « siamoise », comme le montre une collection d'échantillons conservée à la Bibliothèque nationale de France ${ }^{22}$ (fig. 7).
7. Échantillons de « cotonnades ou siamoises ", extraits des Étoffes de Meslay, provenant de la collection du duc de Richelieu (1736), Paris, BnF, département des Estampes et de la Photographie, LH-45-FOL, fol. 83. 


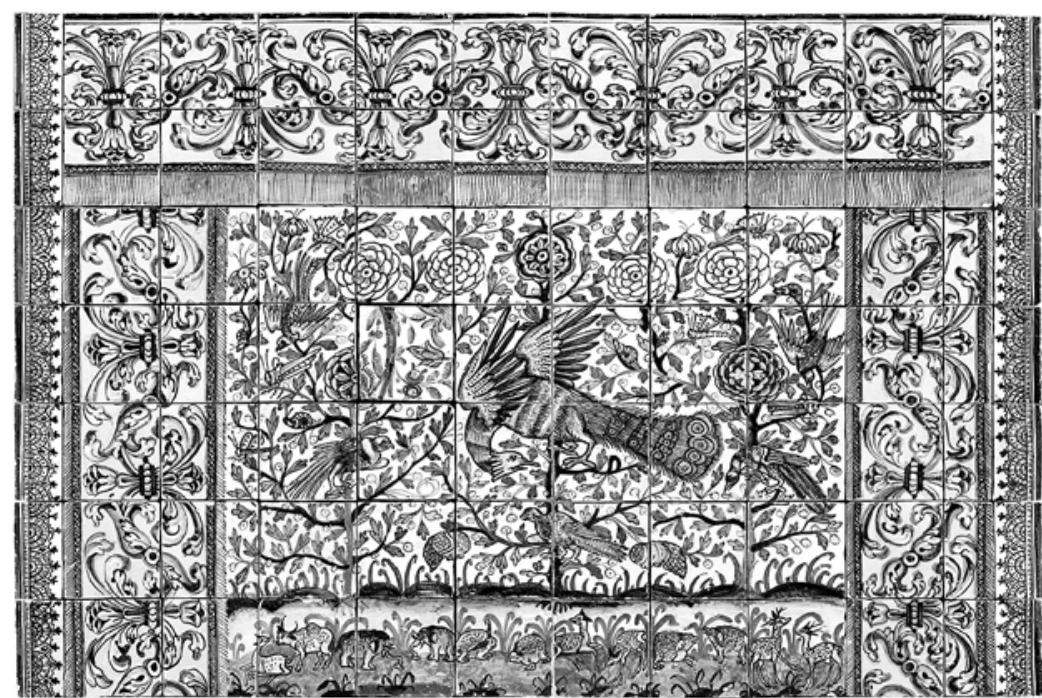

8. Devant d'autel aux oiseaux et aux ramages, azulejos, vers 1625-1650, Lisbonne, Museu Nacional do Azulejo.
Maria João Ferreira. Le fait qu'ils soient facilement transportables et parfaitement adaptables est inhérent à la nature des textiles. À l'instar des gravures, les spécimens textiles constituent de très importantes matrices visuelles dont la diffusion concourt à la création et à la transmission de répertoires ornementaux vraiment transculturels - avec pour avantage $d$ 'offrir un ensemble plus vaste $d^{\prime}$ information dans les domaines de la chromatique et de la technologie, dont ils sont simultanément porteurs.

$\mathrm{Au}$ fil du temps et sous les latitudes géographiques et culturelles les plus diverses, les textiles ont été intégrés, imités et recréés avec différents critères et suivant différents niveaux d'approche selon les participants concernés - artisans, intermédiaires et destinataires (institutionnels ou privés, religieux ou laïques, acquéreurs ou vendeurs) - leurs aspirations et leurs connaissances. Dans ce processus d'apprentissage et de réinterprétation des formes et des motifs, I'influence des textiles comme source d'inspiration et d'enseignement pratique s'étend à d'autres domaines artistiques selon différentes modalités, tant par l'intermédiaire de partenariats, qui s'établissent en termes créatifs et commerciaux, que par le recours à des projets communs - comme le sont les répertoires de dessins - répartis entre les textiles et d'autres types de supports, dont les particularités (comme la bi-dimensionnalité) entraînent des approches similaires.

Aux XVI et XVII siècles, l'influence des textiles est évidente dans le répertoire structurel et thématique des azulejos portugais. Avec d'autres matériaux et d'autres techniques de fabrication, les devants d'autel reproduisent et s'inspirent de la composition des modèles, des détails et du vocabulaire textile contemporain. À côté des référents typiquement occidentaux (européens) se détachent de plus en plus les motifs asiatiques qui, après l'ouverture de la Route du Cap, furent introduits dans le royaume à une échelle sans précédent. Les spécimens venus de l'Inde, de la Perse et de la Chine gagnèrent en prestige dans l'éventail de possibilités qui jusqu'alors constituait le principal marché de l'offre au Portugal ${ }^{23}$ et devinrent d'importantes sources $d^{\prime}$ inspiration pour ce qu'on a appelé les devants d'autel aux oiseaux et aux ramages ${ }^{24}$ (fig. 8). Produite en majorité dans les ateliers lisboètes, surtout entre le deuxième et le troisième quart du XVII siècle, cette typologie est considérée comme l'une des plus riches expressions de la transculturation de l'art portugais du XVII ${ }^{\mathrm{e}}$ siècle, si grand est le syncrétisme qui la caractérise.

Maria Ludovica Rosati. Bien avant le développement des "global studies", les premières recherches sur les textiles et les premières collections témoignaient déjà d'une prise de conscience embryonnaire $d u$ besoin d'aller au-delà des traditionnelles frontières nationales, et s'intéressaient aux productions étrangères. Des orientations critiques récentes ont fourni de nouveaux outils d'approche méthodologique des phénomènes interculturels les plus divers. Si l'on tient compte de sa vocation traditionnelle, 
le champ des textiles au début de l'époque moderne pourrait-il devenir un champ d'investigation propice à l'application des théories transculturelles? Quels avantages pourrait-on en tirer?

Maria João Ferreira. Les frontières de la production et de la consommation des «Trade textiles ${ }^{25}$ » à l'époque moderne se révèlent bien plus fluides que les frontières géopolitiques traditionnellement admises par I'historiographie. La vie des objets atteste un monde dynamique et relié qui interpelle sur la nécessité d'une histoire plus ouverte, capable de refléter ces connexions (quand ce ne sont pas des superpositions) et qui impose des changements d'interprétation tant au niveau de la perception de l'espace et du temps que de l'identité. Le textile d'origine chinoise produit dans le cadre de la présence portugaise en Asie, à partir du XVI e siècle, est symptomatique de cette réalité. $\mathrm{D}^{\prime}$ une part, la Chine a une profonde influence dans les royaumes adjacents qui délimitent la mer de Chine et dans la totalité de l'espace géographique que constitue la région s'étendant entre l'océan Indien et l'océan Pacifique. Ainsi, la démonstration, au sein d'une manufacture donnée, de caractéristiques connotant la production chinoise ne signifie pas pour autant que celle-ci en soit strictement à l'origine - d'autant plus que, outre les objets, les matériaux et surtout les personnes circulent en transportant avec elles les idées et la connaissance. D'autre part, jusqu'à la première moitié du XVII siècle, les Portugais se révélèrent $d^{\prime}$ importants partenaires commerciaux de la Chine : en qualité d'agents intermédiaires, ils pouvaient influer sur le profil des marchandises chinoises négociées. À partir de Macao, ils transportaient les produits textiles chinois vers le Japon (jusqu'en 1639) et vers l'Inde (d'où ils étaient acheminés vers le Portugal et l'Europe) et s'intégraient dans les circuits commerciaux hispano-philippins et américains. Dans ce contexte, les approches transculturelles, interdisciplinaires et multidimensionnelles qui mettent en relation et reconnaissent l'importance de l'économie mais aussi celle de la politique, de la culture et de l'art dans ce phénomène complexe, paraissent indispensables pour une étude efficace des influences qui se développèrent de façon croisée et parallèle tout au long de ces circuits, d'autant plus lorsqu'elles sont articulées avec des travaux exhaustifs d'inventaire d'objets susceptibles de fournir des bases solides pour l'étude des cas spécifiques et des analyses comparatives.

Corinne Thépaut-Cabasset. Quel fut le succès des produits européens dans le Nouveau Monde au début du XVIII siècle ? Quelle méthode doit-on appliquer pour étudier les modes et les textiles européens qui ont transformé ou non la manière de s'habiller dans les colonies espagnoles?

L'impact de la mode sur la culture des apparences dans le Nouveau Monde est très peu connu, et les rares recherches entreprises se limitent aux aspects socioéconomiques. L'étude pionnière que Fernand Braudel a consacrée à I'histoire du monde moderne préindustriel est considérée comme l'un des ouvrages précurseurs de la théorie des systèmes-monde ${ }^{26}$.

Le projet de recherche intitulé "Dressing the New World ${ }^{27}$ » s'appuie sur un document exceptionnel qui décrit le marché de Mexico dans les années 1700. Ce manuscrit de Jean de Monségur ${ }^{28}$ (des mémoires sur la Nouvelle Espagne rédigés dans la première décennie du siècle) constitue l'une des rares références à l'Amérique espagnole au début du XVIII ${ }^{e}$ siècle, et une source précieuse sur la dissémination et la consommation des produits européens de l'autre côté des mers. Le projet a pour objectif $d^{\prime}$ examiner en détail les modes et le textile à travers ce texte et d'autres documents tirés de la littérature commerciale de l'époque, de l'iconographie ou de la culture matérielle, en faisant intervenir différentes disciplines : histoire moderne, histoire de l'art et histoire du costume. Il s'efforce également d'étudier le rôle du textile 


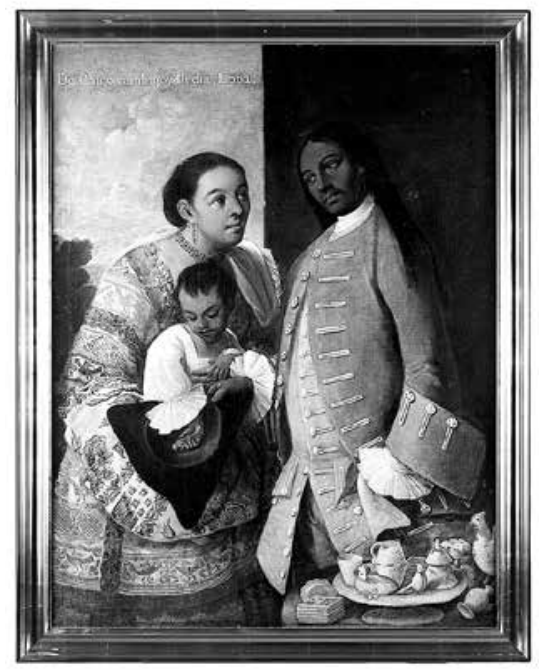

et de la mode dans les études coloniales ${ }^{29}$. Il vise enfin à prendre en compte I'action de la politique et l'importance des liens planétaires dans les études sur le costume et la culture visuelle au début de l'époque moderne.

Les rapports officiels, la correspondance politique et les récits de voyageurs constituent une riche source d'information sur l'histoire des textiles et des modes, leur consommation et leur distribution au cours de la période. Prises ensemble, ces sources fournissent une occasion unique de découvrir le réseau de relations entre différentes régions du monde nourri par les échanges commerciaux à l'époque. Cette recherche vise ainsi à écrire un nouveau chapitre de l'histoire mondiale du costume en s'aventurant dans les récits de voyage, la peinture et les collections de costume conservées dans les musées. Enfin, il s'agit de se demander si les articles de mode et les textiles européens ont fourni ou non l'occasion d'un transfert culturel au Mexique et ailleurs.

En s'appuyant également sur l'exceptionnelle source iconographique que constitue la " peinture de caste ${ }^{30}$ » (fig. 9), ces recherches permettront de mettre au point un premier glossaire illustré des textiles et des costumes commercialisés à l'échelle mondiale à l'époque préindustrielle ${ }^{31}$.

9. Miguel Cabrera, De Chino cambujo e India, Loba, 1763, Madrid, Museo de América.
Liza Oliver. Les frontières disciplinaires sont le plus souvent tracées à partir du concept d'État-nation, ce qui constitue un obstacle majeur au développement des études transculturelles. Le commerce textile à l'époque pré-moderne est un champ d'investigation particulièrement propice au démantèlement de ces frontières, car il s'est structuré en dehors des cadres géographiques et idéologiques instaurés par la modernité et la colonisation. Les routes commerciales terrestres et maritimes relient depuis toujours diverses régions du globe, révélant le caractère artificiel de l'imposition du concept d'État-nation sur la manière dont nous organisons nos connaissances du monde.

Mon optimisme s'accompagne cependant d'une mise en garde concernant essentiellement le mauvais usage qui pourrait être fait de la formule " global studies » et la manière dont on classifie toujours plus les spécialisations géographiques. L'histoire de l'art " européen » reste immuable, tandis que l'histoire de l'art " globale » est devenue le réceptacle de tout ce qui concerne le «non-Occident ». Les " global studies » pourraient ainsi fort bien faire le jeu d'un paternalisme euro-centrique, et réaffirmer les frontières qu'elles prétendent abolir. Dans cette éventualité, elles rétabliraient, sous une autre forme, les catégorisations euro-centriques de l'« Autre ». Elles ne permettraient donc aucunement d'aller au-delà des frontières nationales, et encore moins de les remettre en question.

Le second point d'inquiétude concerne le lien entre local et planétaire lorsqu'il s'agit d'histoire transculturelle. Sur ce terrain, comme l'a souligné Sanjay Subrahmanyam, on se voit contraint de naviguer constamment entre ces deux extrêmes en tension. D'un côté, on risque de minimiser les facteurs planétaires qui influent sur une société locale ; de l'autre, on risque d'octroyer un rôle trop important à ce qui est situé au-delà du cadre de cette société32. Trouver un moyen terme responsable entre les deux requiert de l'expérience et une connaissance approfondie des sociétés considérées dans l'étude; I'histoire mondiale se fonde sur un ensemble d'histoires locales, et non sur l'omission de telle ou telle. Mais tant que notre système universitaire restera prisonnier du cadre de l'État-nation, il sera difficile de mettre au point les compétences et les connaissances requises pour cette tâche.

Cela ne signifie pas pour autant que le champ des textiles n'est pas à la hauteur du défi. II s'agit plutôt de veiller à ne pas dissiper son potentiel méthodologique en laissant par inadvertance celui-ci se fondre dans un usage inconsidéré du terme 
" global ». Pour ma part, j'estime que les « global studies » et les théories transculturelles doivent contribuer au remodelage des structures disciplinaires, et non relever d'une simple substitution entre termes. C'est, à mes yeux, le plus grand avantage méthodologique que puisse apporter l'étude des textiles à l'histoire de l'art en tant que discipline.

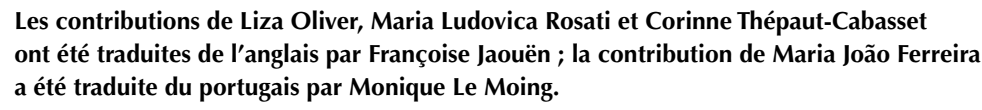

1. Giorgio Riello, Peter McNeil (dir.), The Fashion History Reader: Global Perspective, Londres/New York, 2010 ; The Wor/wide Textile Trade, 1500-1800, Amelia Peck (dir.), cat. exp. (New York, The Metropolitan Museum of Art, 2013-2014), Londres, 2013 ; Marie-Louise Nosch, Zhao Feng, Lotika Varadarajan (dir.), Global Textile Encounters, Oxford/Philadelphie, 2014 ; Luca Molà, Dieter Schäfer (dir.), Threads of Global Desire: Silk in the Pre-Modern World, Oxford [à paraître].

2. Gaspar Correia, Crónicas de D. Manuel e de D. João III (1533), José Pereira da Costa (éd.), Lisbonne, 1992.

3. Voir par exemple le décor et les tapis représentés dans le tableau d'André Reinoso, Saint François-Xavier saluant à Jean III avant son départ pour l'évangélisation de l'Inde, vers 1619, Lisbonne, Museu de São Roque, inv. 94.

4. Lorge M. Flores, "Um Império de Objetos", dans Mafalda S. Cunha (dir.), Os Construtores do Oriente Português, Lisbonne, 1998, p. 15-51, p. 26-27.

5. Voir Philip Wagoner, "Sultan among Hindu Kings": Dress, Titles, and Islamicization of Hindu Culture at Vijayanagara ", dans The Journal of South Asian Studies, 55, 4, 1996, p. 851-880 ; Finbarr Barry Flood, Objects of Translation: Material Culture and the "Hindu-Muslim" Encounter, Princeton, 2009.

6. Christopher Alan Bayly, "The Origins of Swadeshi (Home Industry): Cloth and Indian Society, 1700-1930", dans Arjun Appadurai (dir.), The Social Life of Things: Commodities in Cultural Perspective, Cambridge/New York/New Rochelle, 1986, p. 285-321.

7. Corinne Thépaut-Cabasset, "Présents du Roi: An Archive at the Ministry of Foreign Affairs in Paris", dans Studies in Decorative Arts: An International Journal, XV, 1, automne-hiver 2007-2008, p. 4-18.
8. Eadem, "Newly Discovered Documents Help to Reconstruct the Purchase of a Lost Princely Wardrobe ", dans ICOM-Costume 2015 Proceedings, en ligne: http://network.icom.museum/ fileadmin/user_upload/minisites/costume/pdf/ The__paut-Cabasset_ed_Pietsch.pdf (consulté le 16/06/2016).

9. Eadem, «Diplomatische Agenten und der Europäische Luxuswarenhandel im späten 17. Jahrhundert ", dans Mark Häberlein, Christof Jeggle (dir.), Materielle Grundlagen der Diplomatie: Schenken, Sammeln und Verhandeln in Spätmittelalter und Früher Neuzeit, Constance/Munich, 2013, p. 157-75; Eadem, " Garde-robe de souverain et réseau international : I'exemple de la Bavière dans les années 1680 », dans Isabelle Paresys, Natacha Coquery (dir.), Se vêtir à la cour en Europe (1400-1815), actes de colloque (Versailles, château de Versailles, 2009), Villeneuve d'Ascq, 2011, p. 177-193.

10. Nadia Fernandez-de-Pinedo, Corinne ThépautCabasset, " A Taste for French Style in Bourbon Spain: Food, Drink, and Clothing in 1740s Madrid », dans Jane Stobart (dir.), A Taste for Luxury in Early Modern Europe, Londres, à paraître.

11. Corinne Thépaut-Cabasset, L'Esprit des modes au Grand siècle, Paris, 2010.

12. Nicolas Arnoult, Femme de qualité habillé [sic] en sultane, 1688, Paris, BnF, département des Estampes et de la Photographie, OA-51-PET FOL, fol. 51 (fig. 4).

13. Maria João Ferreira, "Chinese Textiles for Portuguese Taste ", dans The Wor/wide Textile Trade..., 2013, cité n. 1, p. 46-55.

14. Rosemary Crill, "Local and Global: Patronage and Use ", dans The Fabric of India, Eadem (dir.), cat. exp. (Londres, Victoria and Albert Museum, 2015-2016), Londres, 2015, p. 78-179, p. 140.

15. António M. Hespanha, "O Orientalismo em Portugal (séculos XVI-XX) », dans Ana Maria 
Rodrigues (dir.), O Orientalismo em Portugal, Lisbonne, 1999, p. 15-37, p. 20-21.

16. Paul Gilroy, The Black Atlantic Modernity and Double-Consciousness, Cambridge [Mass.], 1993 [éd. fra. : L'Atlantique noir : modernité et double conscience, Lille/Paris, 2003].

17. Pour une analyse plus détaillée, voir Beth Fowkes Tobin, " Taxonomy and Agency in Brunias's West Indian Paintings ", dans Picturing Imperial Power: Colonial Subjects in 18th-Century British Painting, Durham/Londres, 1999, p. 139-173.

18. Corinne Thépaut-Cabasset, "Fashion Encounters: The "Siamoise", or the Impact of the Great Embassy on Textile Design in Paris in 1687 ", dans Nosch, Feng, Varadarajan, 2014, cité n. 1, p. 18.

19. Mercure Galant, septembre 1686 - janvier 1687.

20. Thépaut-Cabasset, 2010, cité n. 11, p. 30.

21. Voir Nicolas Arnoult, Femme de qualité en habit d'été d'étoffe siamoise, Paris, BnF, département des Estampes et de la Photographie, OA-62PET FOL, fol. 76 .

22. Voir également, entre autres, les "Siamoises" de la manufacture de Rouen, issues de la même collection d'étoffes du duc de Richelieu (1737), Paris, BnF, département des Estampes et de la Photographie, LH-45-FOL.

23. Maria João Ferreira, "Asian Textiles in the Carreira da India: Portuguese Trade, Consumption and Taste, 1500-1700 ", dans Textile History, 46, 2, novembre 2015, p. 147-168.

24. Voir l'exemplaire conservé par le Museu Nacional do Azulejo, à Lisbonne, inv. $132 \mathrm{Az}$ (fig. 8).

25. Amelia Peck, "Trade Textiles at the Metropolitan Museum: A History ", dans The Worlwide Textile Trade..., 2013, cité n. 1, p. 2-11, p. 9.

26. Fernand Braudel, Civilisation matérielle, économie et capitalisme: XV-XVIII siècles (1967), Paris, 1979 [pour l'édition en trois volumes : I. Les Structures du quotidien, le possible et l'impossible; II. Les Jeux de l'échange; III. Le Temps du monde].

27. "Dressing the New World: The Trade and Culture of Clothing in the New Spanish Colonies 1600-1800 », projet mené par C. Thépaut-Cabasset, au Centre de recherche sur les textiles (CTR) à I'université de Copenhague, avec le soutien d'une bourse Marie Skłodwska-Curie (Horizon 2020).

28. Jean-Paul Duviols, Mémoires du Mexique. Le Manuscrit de Jean de Monségur (1707-1709), Paris, 2002

29. Rebecca Earle, The Body of the Conquistador: Food, Race, and the Colonial Experience in Spanish America, Cambridge, 2012 ; Serge Gruzinski, The Mestizo Mind: The Intellectual Dynamic of Colonization and Globalization, New York, 2002 [éd. orig. La Pensée métisse, Paris, 1999]; Dana
Leibsohn, Jeanette Favrot-Peterson (dir.), Seeing Across Cultures in the Early Modern World, Farnham/Burlington [Vt], 2012.

30. Ilona Katzew, Casta Paintings: Images of Race in Eighteenth-Century Mexico, New Haven, 2004 ; Painting a New World: Mexican Art and Life, 15211821, Donna Pierce, Rogelio Ruiz Gomar, Clara Bargellini (dir.), cat. exp. (Denver, Denver Art Museum, 2004), Denver, 2004 ; Magali M. Carrera, Imagining Identity in New Spain: Race, Lineage, and the Colonial Body in Portraiture and Casta Paintings, Austin, 2003.

31. Voir également, entre autres, Miguel Cabrera, De español y mestiza, 1763, Madrid, Museo de América, inv. 00006.

32. Sanjay Subrahmanyam, Merchants, Markets, and the State in Early Modern India, Delhi, 2008, p. 8 . 